\title{
Combining 4D Flow MRI and Complex Networks Theory to Characterize the Hemodynamic Heterogeneity in Dilated and Non-dilated Human Ascending Aortas
}

\author{
Karol Calò, ${ }^{1}$ Diego Gallo, ${ }^{1}$ Andrea Guala, ${ }^{2,3}$ Jose Rodriguez Palomares, $, 2,3,4$ \\ Stefania Scarsoglio, ${ }^{1}$ Luca Ridolfi, ${ }^{1}$ \\ and Umberto Morbiducci (iD ${ }^{1}$
}

${ }^{1}$ PolitoBIOMed Lab, Department of Mechanical and Aerospace Engineering, Politecnico di Torino, Corso Duca degli Abruzzi 24, 10129 Turin, Italy; ${ }^{2}$ Vall d'Hebron Institut de Recerca (VHIR), Barcelona, Spain; ${ }^{3}$ CIBER-CV, Instituto de Salud Carlos III (ISCIII), Madrid, Spain; and ${ }^{4}$ Department of Cardiology, Hospital Universitari Vall d'Hebron, Barcelona, Spain

(Received 15 March 2021; accepted 17 May 2021; published online 2 June 2021)

Associate Editor Stefan M Duma oversaw the review of this article.

\begin{abstract}
Motivated by the evidence that the onset and progression of the aneurysm of the ascending aorta (AAo) is intertwined with an adverse hemodynamic environment, the present study characterized in vivo the hemodynamic spatiotemporal complexity and organization in human aortas, with and without dilated AAo, exploring the relations with clinically relevant hemodynamic and geometric parameters. The Complex Networks (CNs) theory was applied for the first time to 4D flow magnetic resonance imaging (MRI) velocity data of ten patients, five of them presenting with AAo dilation. The time-histories along the cardiac cycle of velocity-based quantities were used to build correlationbased CNs. The CNs approach succeeded in capturing largescale coherent flow features, delimiting flow separation and recirculation regions. CNs metrics highlighted that an increasing AAo dilation (expressed in terms of the ratio between the maximum AAo and aortic root diameter) disrupts the correlation in forward flow reducing the correlation persistence length, while preserving the spatiotemporal homogeneity of secondary flows. The application of CNs to in vivo 4D MRI data holds promise for a mechanistic understanding of the spatiotemporal complexity and organization of aortic flows, opening possibilities for the integration of in vivo quantitative hemodynamic information into risk stratification and classification criteria.
\end{abstract}

Keywords-Ascending aorta aneurysm, Aortic dilation, Magnetic resonance imaging, Network science, Spatiotemporal analysis.

Address correspondence to Umberto Morbiducci, PolitoBIOMed Lab, Department of Mechanical and Aerospace Engineering, Politecnico di Torino, Corso Duca degli Abruzzi 24, 10129 Turin, Italy. Electronic mail: umberto.morbiducci@polito.it

\section{INTRODUCTION}

Ascending aorta (AAo) aneurysm is a dilation of the segment of the aorta proximal to the brachiocephalic trunk. To avoid life-threatening complications such as dissection and rupture, AAo aneurysms are repaired by elective surgery, recommended mainly on the basis of the maximum diameter (with a fixed threshold of $5.5 \mathrm{~cm}$ in the vast majority of cases). ${ }^{10,38}$ However, surgical repair is associated with significant mortality rates $(3-5 \%)^{27}$ and aortic diameter alone has proved to be an ineffective predictor of events. ${ }^{15,38}$ These aspects have motivated research on mechanisms behind AAo aneurysm evolution as well as on new criteria for risk stratification. Although AAo dilation is the result of a multifactorial process involving genetics expressions, biological and structural factors, ${ }^{15,48}$ there is evidence of the role played by local adverse hemodynamics in flow-mediated mechanisms leading to adverse vascular remodeling. ${ }^{24,39}$ In particular, hemodynamics in dilated AAo is characterized by abnormal blood flow that reflects in near-wall flow disturbances ${ }^{3,6,43,44}$ leading to mechanical alterations in the aortic wall. ${ }^{6,11,13,23,26}$ Furthermore, hemodynamic disturbances are intertwined with and exacerbated by concomitant AAo dilation and aortic valve abnormalities, such as bicuspid aortic valve (BAV) or deficient tricuspid aortic valves (TAV). ${ }^{14,28,36,46}$ 
In recent years, 4D flow magnetic resonance imaging (MRI) has been increasingly used to obtain information on both aortic morphology and hemodynamics $5,14,31,35,43$ - in particular in the presence of aortic vascular/valve pathologies ${ }^{4,16,23,31}$ —providing risk markers of AAo wall degeneration. ${ }^{14,25,43}$

With the objective of providing a comprehensive characterization of the spatiotemporal heterogeneity of large-scale aortic flow features and of their possible links with AAo dilation, a recently proposed approach integrating computational hemodynamics with Complex Networks (CNs) theory ${ }^{7,8,13,37}$ is here extended for the first time to 4D flow MRI in patients with and without aortic dilation. In this study we explored the capability of $\mathrm{CNs}$ to characterize in vivo the dynamics of dominant aortic flow features using the time-histories of the measured velocity data along the cardiac cycle. Patient-specific CNs were built with MRI voxels belonging to the aortic fluid domain as nodes of the networks, which were connected by links based on the strength of the pairwise correlation between velocity time-histories. CNs metrics allowed for the evaluation of the anatomical and topological length of correlation persistence of velocity time-histories within the aorta and its association with clinically relevant hemodynamic and geometric parameters. In prospect, the application of $\mathrm{CNs}$ can lead to a deeper understanding of the factors and basic mechanisms influencing the spatiotemporal complexity of aortic flows. The improved characterization of the disease has the potential to strengthen the clinical utility of blood flow visualizations, enhance diagnostic strategies and tools in terms of possible application to risk stratification and classification criteria, and inform clinical decisionmaking.

\section{MATERIALS AND METHODS}

Ten patients were enrolled for this study, five of them presenting with AAo dilation (one of them with bicuspid aortic valve, BAV), and five without AAo dilation (one with BAV). ${ }^{14}$ Ascending aortic dilation was diagnosed according to the protocol proposed elsewhere. ${ }^{9}$ Briefly, ascending aortic dilation was defined as at least 1.96 standard deviations above the normal diameter for a specific patient either at the aortic root or at the AAo, at the level of the pulmonary artery bifurcation, using previously proposed reference values. ${ }^{9}$ All patients presented with mild to severe aortic valve dysfunction (Table 1). The study was approved by the ethics committee of the Vall d'Hebron Hospital and informed consent was obtained from all participants.

\section{D FLOW MRI ACQUISITION AND DATA PROCESSING}

All patients underwent 4D flow MRI acquisitions of the entire thoracic aorta with retrospective ECG gating during free-breathing and no endovenous contrast agent. Briefly, a radially undersampled acquisition with five-point balanced velocity encoding ${ }^{29}$ was used. Phase-contrast MRI acquisitions were set according to the following scheme: velocity encoding in the range of $150-400 \mathrm{~cm} \mathrm{~s}^{-1}$, field of view $400 \times 400 \times 400 \mathrm{~mm}$, scan matrix $160 \times 160 \times 160$, voxel size $2.5 \times 2.5 \times 2.5 \mathrm{~mm}$, and number of cardiac frames in the range of 30-46. Full details on the adopted 4D flow MRI acquisition protocol are reported in previous studies. ${ }^{14,29}$

Lumen segmentation of the thoracic aortas was performed on phase-contrast enhanced MR angiogram using ITK-Snap and used for a centerline-based reconstruction of 3D aortic geometries in VMTK ${ }^{1}$ (www.vmtk.org). Anatomic landmarks were identified from co-registered 2D cine images and used to ensure a consistent spatial extent across all patients, ${ }^{8,21}$ defining the AAo, the aortic arch, and the descending aorta (DAo) portions used for the CNs analysis (Fig. 1). The reconstructed $3 \mathrm{D}$ geometries were used as masks for the acquired velocity data. ${ }^{47}$

\section{Quantitative Hemodynamic Descriptors}

The acquired phase-velocity data were used to describe the spatiotemporal heterogeneity of large-scale dominant aortic flow features. The time-histories along the cardiac cycle of three intravascular hemodynamic quantities (Fig. 1) were derived from in vivo data: (1) the local velocity magnitude $|\mathbf{V}|$; (2) the axial component $V_{\mathrm{ax}}$ of the local velocity vector $\mathbf{V}$ (or local "through-plane" velocity), defined as the projection of $\mathbf{V}$ along the direction of the tangent to the local vessel centerline ${ }^{8,33}$; (3) the secondary component $V_{\mathrm{sc}}$ of the local velocity vector (or local "in-plane" velocity), orthogonal to the local axial direction and related to secondary flows. ${ }^{33}$ A positive value of $V_{\text {ax }}$ indicates forward flow (along the main, i.e., proximal-to-distal, flow direction), whereas a negative value is representative of retrograde flow. ${ }^{8}$ Similarly, positive and negative values of $V_{\text {sc }}$ indicate in-plane right- and lefthanded direction, respectively, when viewed in the direction of the forward movement. ${ }^{33}$

Geometric and hemodynamic quantities measurable in a clinical framework were also evaluated from 4D flow MRI data (Fig. 1) and will be referred to as "clinical parameters" from now on. In detail, the ratio between the maximum AAo diameter $D_{\max }$ (distal to the aortic root), a largely adopted clinical indicator of 
TABLE 1. Aortic dilation and valve type and functional classification of the included patients.

\begin{tabular}{|c|c|c|c|c|}
\hline & Patient & TAV/BAV & Aortic valve insufficiency & Aortic valve stenosis \\
\hline \multirow[t]{5}{*}{ Non-dilated AAo } & $A$ & BAV & Severe & Absent \\
\hline & B & TAV & Moderate & Severe \\
\hline & $\mathrm{C}$ & TAV & Absent & Moderate \\
\hline & $\mathrm{D}$ & TAV & Severe & Absent \\
\hline & $E$ & TAV & Mild & Severe \\
\hline \multirow[t]{5}{*}{ Dilated AAo } & $\mathrm{F}$ & TAV & Mild & Absent \\
\hline & $G$ & TAV & Mild & Absent \\
\hline & $\mathrm{H}$ & BAV & Mild & Severe \\
\hline & $J$ & TAV & Mild & Absent \\
\hline & $\mathrm{K}$ & TAV & Severe & Absent \\
\hline
\end{tabular}

aortic dilation, ${ }^{17}$ and the aortic root maximum diameter $D_{\text {root }}$ was measured and indicated with $D_{\text {ratio. }}$. Moreover, the acquired phase-velocity data were used to quantify the AAo systolic flow eccentricity expressed in terms of flow jet angle (FJA) and normalized flow displacement (FD), as defined elsewhere. ${ }^{45}$ Pulse wave velocity (PWV), considered the gold standard for measuring arterial stiffness, ${ }^{17}$ was non-invasively quantified from 4D flow MRI data in the AAo using a wavelet-based method described elsewhere. ${ }^{2,23}$ As a measure of the energy associated with the largescale aortic flow features, cycle-average and peak blood flow kinetic energy (KE) were computed as:

$$
\mathrm{KE}_{\text {avg }}=\frac{1}{V} \int_{V} \frac{1}{2} \rho|\mathbf{V}|_{\text {avg }}^{2} d V=\frac{1}{V} \int_{V} \frac{1}{2} \rho\left[\frac{1}{T} \int_{0}^{T}|\mathbf{V}(t)| d t\right]^{2} d V
$$

and

$$
\mathrm{KE}_{\text {peak }}=\frac{1}{V} \int_{V} \frac{1}{2} \rho|\mathbf{V}|_{\text {peak }}^{2} d V
$$

respectively. Both $\mathrm{KE}_{\mathrm{avg}}$ and $\mathrm{KE}_{\text {peak }}$ quantities are averaged over the aortic volume $V$. In Eqs. (1) and (2), $\rho$ is the blood density, $|\mathbf{V}|_{\text {avg }}$ is the cycle-average magnitude of the velocity vector $\mathbf{V}(t), T$ is the period of the cardiac cycle, and $|\mathbf{V}|_{\text {peak }}$ is the magnitude of the velocity vector at peak systole.

Finally, a centerline-based analysis was also carried out to obtain aortic mean curvature $(\bar{\kappa})$ and torsion $(\bar{\tau}) .{ }^{41}$

\section{In Vivo CNs Analysis}

The spatiotemporal heterogeneity of the aortic intravascular flow was investigated by using 4D flow MRI data to build patient-specific correlation-based $\mathrm{CNs}$, according to the scheme recently applied to in silico data. ${ }^{7,8,13}$ For each patient, three CNs were built from $|\mathbf{V}|, V_{\text {ax }}$ and $V_{\text {sc }}$ time-histories along the cardiac cycle. Each node of a $\mathrm{CN}$ was defined by the voxel belonging to the aortic fluid domain where the timehistory of the considered hemodynamic quantity was acquired. Two nodes $i$ and $j$ were considered to be connected by a topological link $\{i, j\}$ if the Pearson correlation coefficient $\mathrm{R}_{i j}$ between time-histories at those nodes was greater than a threshold value $\hat{R}$ (Fig. 1). In this study, the threshold values adopted for the construction of the three CNs were selected based on a dataset of computational hemodynamic models of healthy human aortas. ${ }^{13,20,33,34}$ In detail, the median values of the distributions of the correlation coefficients among simulated time-histories were adopted as threshold values for in vivo CNs construction, resulting in $\hat{R}=0.87$ for $|\mathbf{V}|, \hat{R}=0.82$ for $V_{\text {ax }}$, and $\hat{R}=0.03$ for $V_{\mathrm{sc}}\left(V_{\mathrm{sc}}\right.$ correlation coefficients symmetrically distributed around zero). Based on these thresholds, each correlation matrix was converted into an adjacency matrix according to the criterion:

$$
\mathrm{A}_{i j}=\left\{\begin{array}{c}
0, \text { if } \mathrm{R}_{i j} \leq \hat{R} \text { or } i=j, \\
1, \text { if } \mathrm{R}_{i j}>\hat{R} .
\end{array}\right.
$$

Matrix $\mathrm{A}_{i j}$ contains the information on nodes connections: $\mathrm{A}_{i j}=1$ (i.e., nodes $i$ and $j$ are connected by a link) if $\mathrm{R}_{i j}>\hat{R}$, and $\mathrm{A}_{i j}=0$ elsewhere (Fig. 1). For each patient, CNs metrics were applied to characterize the topological structure of $|\mathbf{V}|, V_{\text {ax }}$ and $V_{\text {sc }}$ networks (Fig. 1). The first metric, the hemodynamic degree centrality $h D C_{i}$ is defined as:

$$
h D C_{i}=\frac{1}{N} \sum_{i=1}^{N} \mathrm{~A}_{i j},
$$

where $N$ is the number of voxels in the aorta. The quantity $h D C_{i}$ measures the degree of homogeneity/ heterogeneity of the velocity time-histories acquired at each voxel belonging to the $\mathrm{CN}$ with respect to the whole investigated fluid domain. Technically, it represents the number of nodes of the $\mathrm{CN}$ connected to 


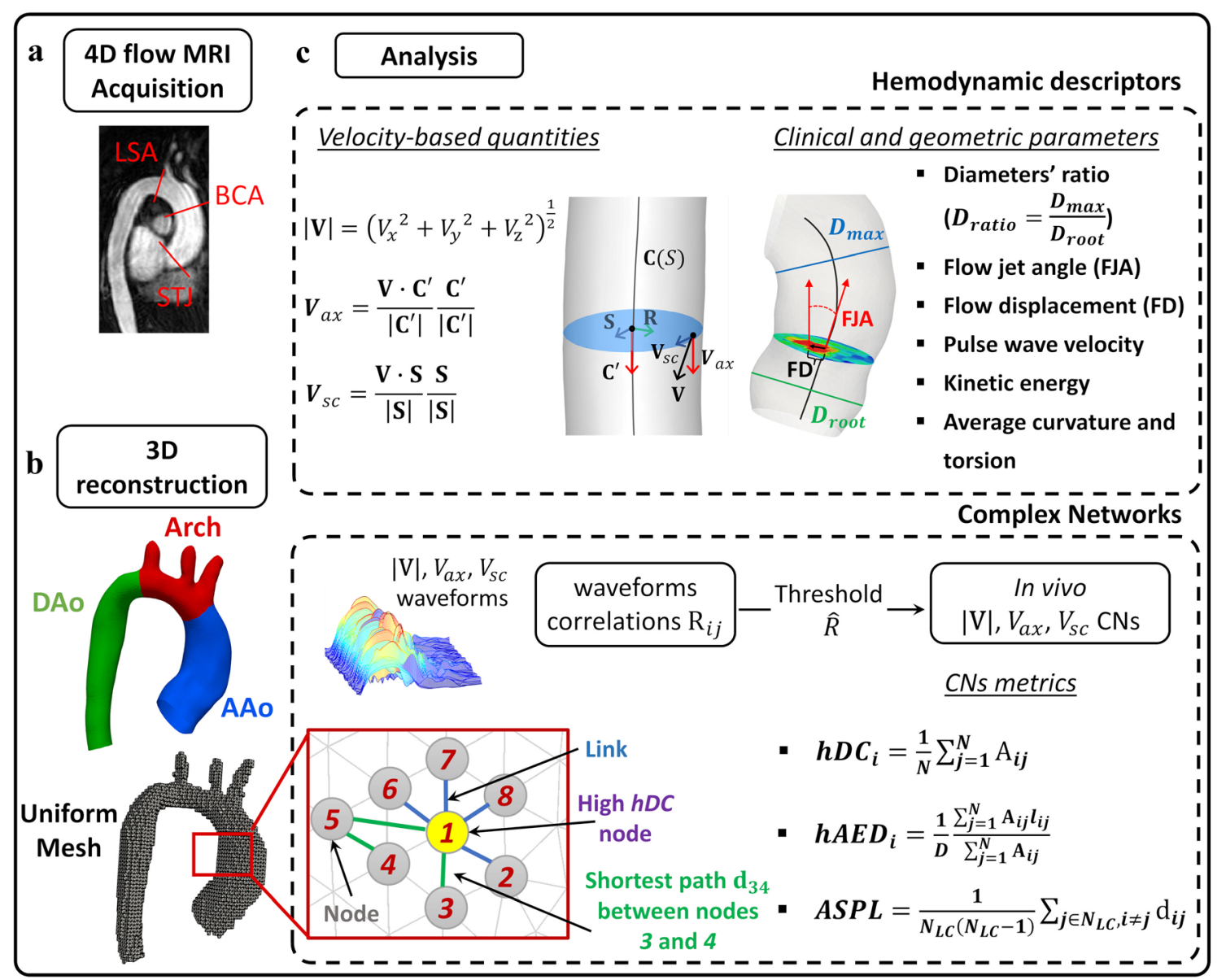

FIGURE 1. Schematic diagram of the methodology used to apply the Complex Networks theory to in vivo velocity data. a: Image acquisition and anatomic landmarks identification from 4D flow MRI: STJ: sinotubular junction; BCA brachiocephalic artery; LSA left subclavian artery. b: Lumen 3D geometry reconstruction. C: Definition of hemodynamic descriptors and complex networks analysis. Hemodynamic descriptors panel: $V_{x}, V_{y}$ and $V_{z}$ : cartesian components of the velocity vector V; C(S): vessel centerline; C': vector tangent to the centerline; R: vector orthogonal to C' directed from the centerline to a generic voxel; S: vector orthogonal to vectors $\mathbf{R}$ and $C^{\prime}$. The investigated clinical and geometric parameters are also listed, with a schematic representation of flow jet angle (FJA), flow displacement (FD), the AAo maximum diameter $D_{\max }$ and the aortic root maximum diameter $D_{\text {root }}$, the latter used to compute $D_{\text {ratio. }}$. Complex Networks panel: $N$ : number of voxels in the aortic domain; $I_{i j}$ : Euclidean distance between voxels $i$ and $j ; N_{\mathrm{LC}}$ : number of nodes of the network's largest connected component, i.e., the maximal set of nodes such that each pair of nodes is connected by a path. An explanatory example of a network is shown, where node 1 is characterized by high hemodynamic degree centrality, the shortest path between nodes 3 and 4 is in green while the other links in blue.

node $i$ (the so-called nearest neighbors of $i$ ), expressed as the percentage of the $N$ voxels of the considered domain. Voxels where $h D C_{i}=0$ were not considered as $\mathrm{CNs}$ nodes because they had no connections with the rest of the network.

The other two CNs metrics provide a quantitative measure of the length of persistence of the correlation between velocity data time-histories. ${ }^{8,13}$ The hemodynamic normalized average Euclidean distance $h A E D_{i}$ (Fig. 1) is defined as:

$$
h A E D_{i}=\frac{1}{D} \frac{\sum_{j=1}^{N} \mathrm{~A}_{i j} l_{i j}}{\sum_{j=1}^{N} \mathrm{~A}_{i j}},
$$

where $l_{i j}$ is the Euclidean distance between node $i$ and its neighbor $j$. To account for aortic geometric variability, $h A E D_{i}$ was normalized with respect to a reference diameter $D$ (Table S1 of the Supplementary Data) identified for each patient as the AAo diameter of a representative healthy subject with the same age, gender and BSA. ${ }^{12}$ High $h A E D_{i}$ values indicate that the correlation between the phase velocity-based timehistory at node (voxel) $i$ and time-histories at its nearest neighbors persists for a large anatomical distance, while low $h A E D_{i}$ values indicate that all the connections of node $i$ are confined to nodes in a small neighborhood. The shortest path length $\mathrm{d}_{i j}$, i.e. the minimum number of links separating two generic nodes $i$ and $j$ of the network, was used to calculate the topological distance metric average shortest path length $A S P L$ of the network ${ }^{8,37}$ (Fig. 1), defined as: 


$$
A S P L=\frac{1}{N_{L C}\left(N_{L C}-1\right)} \sum_{j \in N_{L C}, i \neq j} \mathrm{~d}_{i j},
$$

where $N_{\mathrm{LC}}$ is the number of nodes of the network's largest connected component, i.e., the maximal set of nodes such that each pair of nodes is connected by a path. Based on Eq. (6), ASPL is the average length of the shortest paths (i.e., sequence of consecutive links) in the largest connected network component. High $A S P L$ values indicate that the connections inside the $\mathrm{CN}$ are sparse and that the correlation between the phase velocity-based time-histories persists for a short topological distance.

Linear regressions were used to identify relationships between clinical parameters and $\mathrm{CN}$ metrics. The quality of the regression was evaluated with the Pearson's correlation coefficient R. Significance was assumed for $p<0.05$.

\section{RESULTS}

\section{In Vivo Hemodynamic Analysis}

An overview of the large-scale aortic flow patterns in the ten patients object of this study is presented in Fig. 2 by means of instantaneous streamlines at peak systole. Streamlines visualization highlights a more intricate hemodynamics in dilated patients. Patients with BAV (A and $\mathrm{H}$ ), those affected by valvular dysfunctions (B, C, D, E and K), and dilated AAo patient $\mathrm{J}$ exhibit an eccentric valvular outflow jet impinging on the outer curvature of the AAo (Fig. 2).

The patient-specific analysis of the correlation coefficients $\mathrm{R}_{i j}^{|\mathbf{V}|}, \mathrm{R}_{i j}^{a x}$ and $\mathrm{R}_{i j}^{s c}$ between all pairs of the three velocity-based hemodynamic quantities $|\mathbf{V}|, V_{\text {ax }}$ and $V_{\mathrm{sc}}$ time-histories, respectively, is presented in Figure S1 of the Supplementary Data. The strongest correlations are registered between $|\mathbf{V}|$ time-histories, whereas $V_{\text {ax }}$ correlation coefficients present lower median values due to the presence of anti-correlated waveforms (i.e., negative $\mathrm{R}_{i j}^{a x}$ values). The correlation between $V_{\text {sc }}$ time-histories is symmetrically distributed around the median, which is close to zero for all the patients. Overall, no marked differences can be appreciated between AAo non-dilated and dilated patients (Figure S1 of the Supplementary Data).

\section{In Vivo Complex Networks Analysis}

Volumetric maps of $h D C$ (Fig. 3) highlight the spatiotemporal heterogeneity of the aortic blood flow. Most patients present with scarcely connected networks (low $h D C$ ), reflecting from scarce (as in dilated patients $\mathrm{H}, \mathrm{J}$ and K, Fig. 3) to moderate homogeneity of $|\mathbf{V}|$ and $V_{\text {ax }}$ time-histories. On the other hand, in patient A (Fig. 3) a dense pattern of connections between nodes (voxels) can be observed in the entire aortic domain ( $h D C$ values around $50 \%$ ), indicating an overall high degree of similarity of velocity-based waveforms. In general, the aortic fluid domain is more connected in terms of $V_{\text {ax }}$ than $|\mathbf{V}|$ and two dynamically-distinct regions in terms of $V_{\text {ax }}$ waveforms can be identified: the first one in the DAo, where $V_{\text {ax }}$ waveforms present moderate $h D C$ values, and the second one at the AAo inner wall and aortic arch, where $V_{\mathrm{ax}}$ waveforms present low $h D C$ values. The topologically isolated flow structure identified at the AAo inner wall by close-to-zero $h D C$ values (Fig. 3) highlights the capability of the CNs approach in capturing large-scale blood flow disturbances: at the AAo inner wall the shape of $V_{\text {ax }}$ time-histories along the cardiac cycle is markedly different from the overall aortic hemodynamics. This is the consequence of the combined effect of AAo curvature and aortic valve flow eccentricity, concurring to generate flow separation and recirculation at the inner wall, which in turn interact at the interface with the valve jet shear layer.

Denser patterns of connection between voxels characterize $V_{\text {sc }}$ with respect to $|\mathbf{V}|$ and $V_{\text {ax }}$ time-histories, indicating that $V_{\mathrm{sc}} \mathrm{CNs}$ are more spatiotemporally compact than $|\mathbf{V}|$ and $V_{\mathrm{ax}} \mathrm{CNs}$ and are characterized by the absence of topologically isolated regions ( $h D C$ higher than $40 \%$ in general, Fig. 3). No marked differences emerged between dilated and nondilated patients (Fig. 3).

By visual inspection of the volumetric maps of $h A E D$, quantifying the length of persistence of the correlation in the fluid domain (Fig. 4), in all patients $|\mathbf{V}|$ and $V_{\text {ax }}$ time-histories located close to the AAo inner wall, where typically flow reversal occurs, are characterized by low $h A E D$ values. In the outer region of the AAo and in the Dao, $|\mathbf{V}|$ and $V_{\text {ax }}$ networks are characterized by a neighborhood expanding on longer anatomical distances, confirming the persistence of more correlated flow structures in those regions (Fig. 4).

Figure 5 quantitatively confirms the observation that in general the anatomical length of correlation persistence: (1) is longer in $V_{\text {ax }}$ networks than in $|\mathbf{V}|$ networks; (2) in $V_{\text {ax }}$ and $|\mathbf{V}|$ networks it is shorter in dilated compared to non-dilated patients; (3) in both $V_{\text {ax }}$ and $|\mathbf{V}|$ networks it is shorter than two reference diameters $D(|\mathbf{V}|$ median values $=1.74 D$ and $0.89 D$, and $V_{\text {ax }}$ median values $=1.95 \mathrm{D}$ and $1.65 \mathrm{D}$, for nondilated and dilated patients, respectively). On the opposite, both dilated and non-dilated $V_{\text {sc }}$ networks are characterized by an anatomical length of correlation persistence longer than two reference diameters $D$ (median values $=2.13 \mathrm{D}$ and $2.41 \mathrm{D}$, for non-dilated 


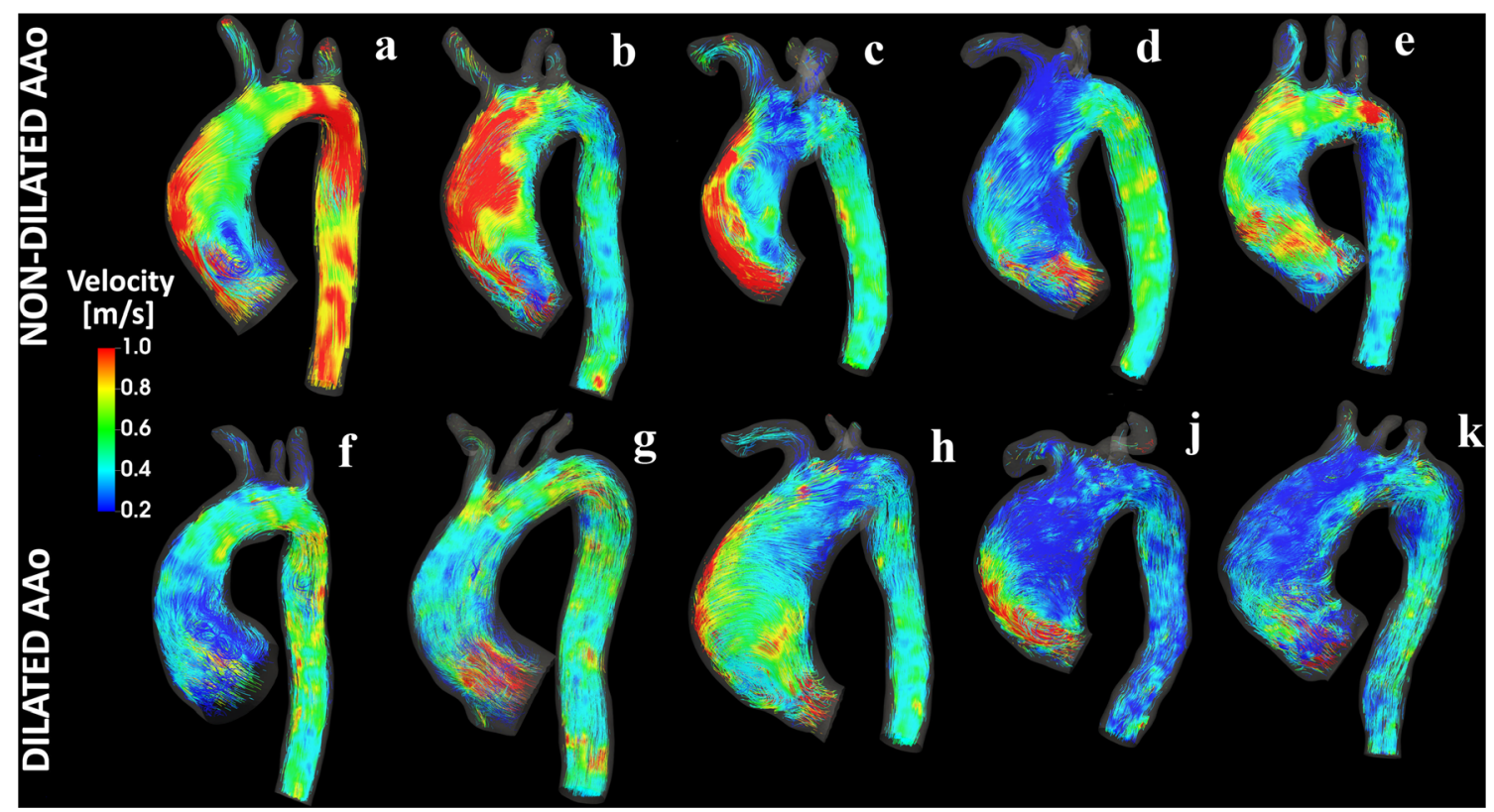

FIGURE 2. Visualizations of instantaneous streamlines at peak systole in patients with (bottom row) and without (top row) ascending aortic dilation. Colors represent velocity magnitude.
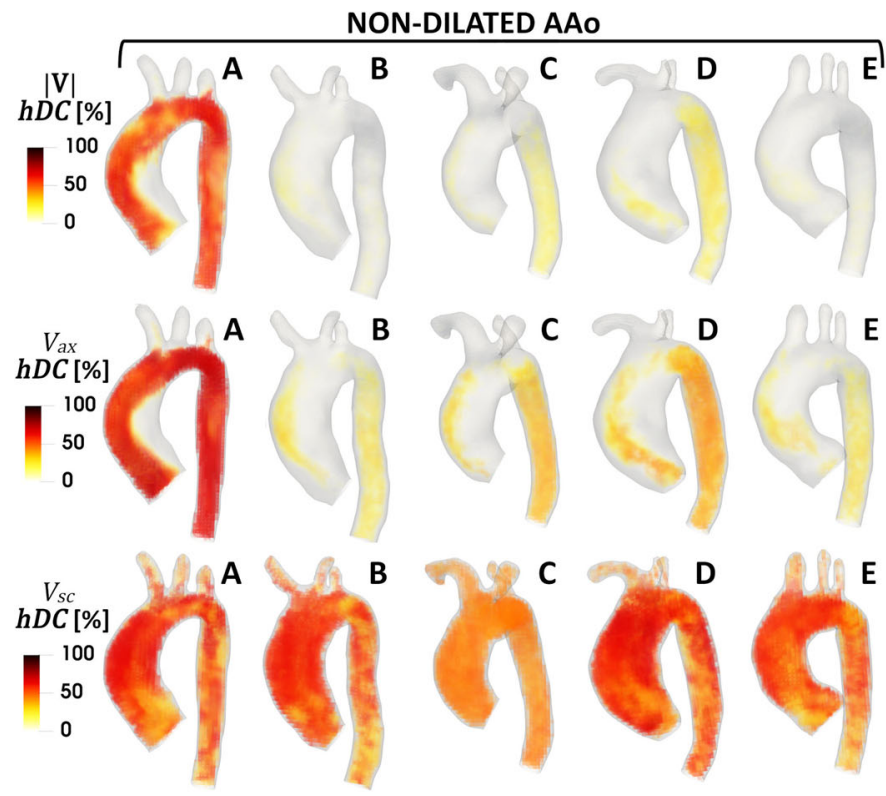

C
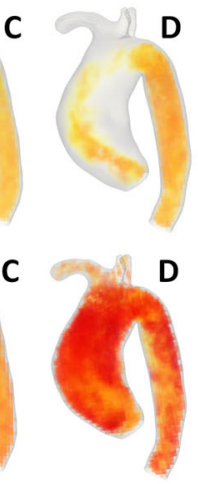

$\mathbf{E}$
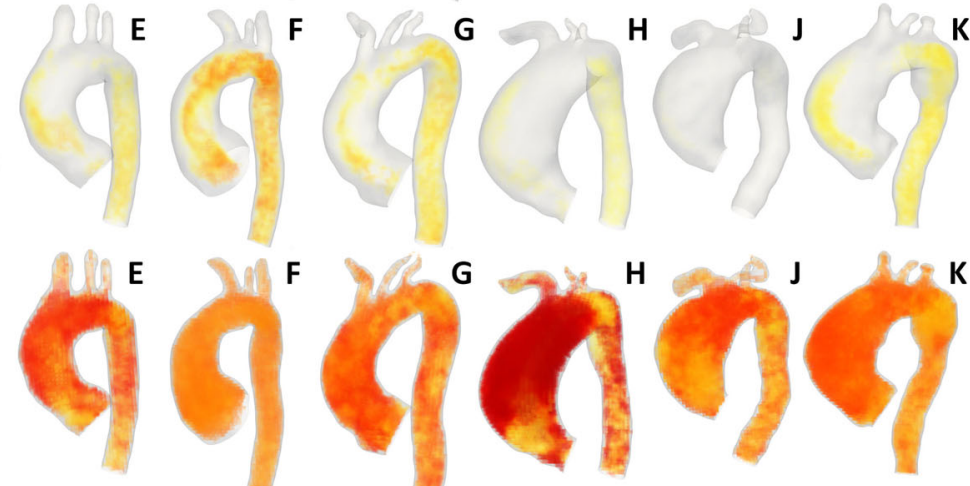
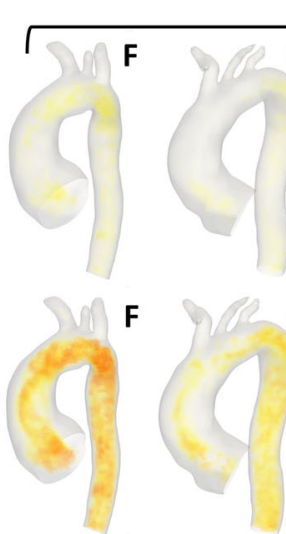

$\mathbf{G}$

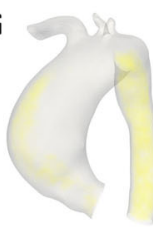

H

DILATED AAO
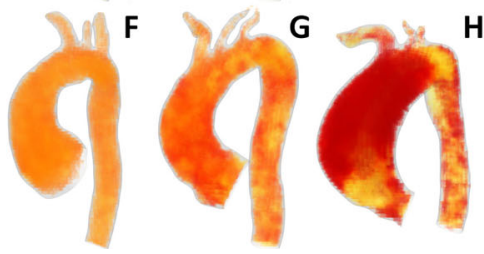

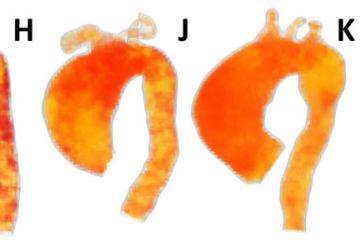

FIGURE 3. Volumetric maps of $h D C$ for IVI (top row), $V_{\text {ax }}$ (middle row) and $V_{\text {sc }}$ (bottom row) CNs.

and dilated patients, respectively). Moreover, non-dilated patients present with median $h A E D$ shorter than dilated ones (Fig. 5), suggesting a major contribution for AAo dilation in shaping secondary blood flow patterns.

The analysis of the topological separation of the largest connected fluid structures in the aortic fluid domain, as quantified by the metric $A S P L$ (Table 2), confirms the findings of the anatomy-based investiga- tion summarized in Figs. 4 and 5. In detail, it emerges that in general two generic voxels of the largest component of the $|\mathbf{V}|$ networks are separated by a median of 3.05-links path in non-dilated patients, and by a 4.83-links path in dilated patients (Table 2), suggesting a role for AAo dilation in breaking up topological links. Although with a less marked difference, median $A S P L$ values in the largest connected components of the $V_{\text {ax }}$ networks reflect the results of the $|\mathbf{V}|$ networks

\section{BMES}




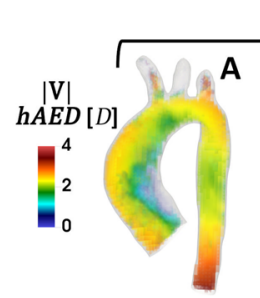

\section{NON-DILATED AAO}
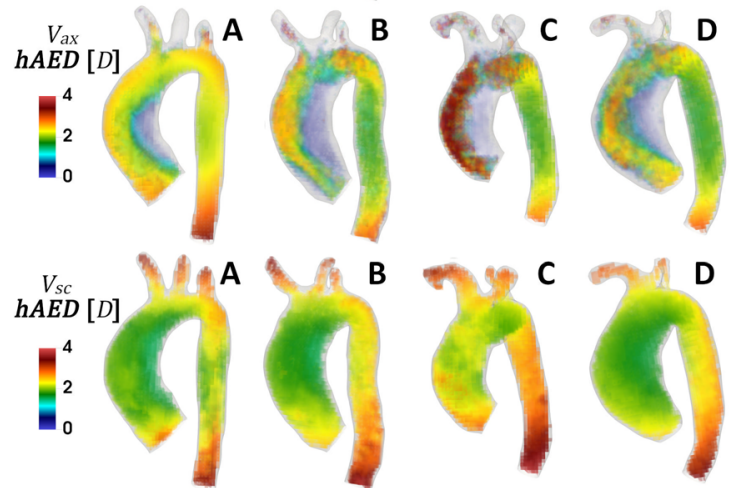

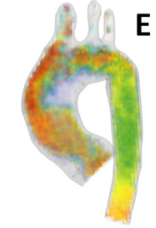

DILATED AAO
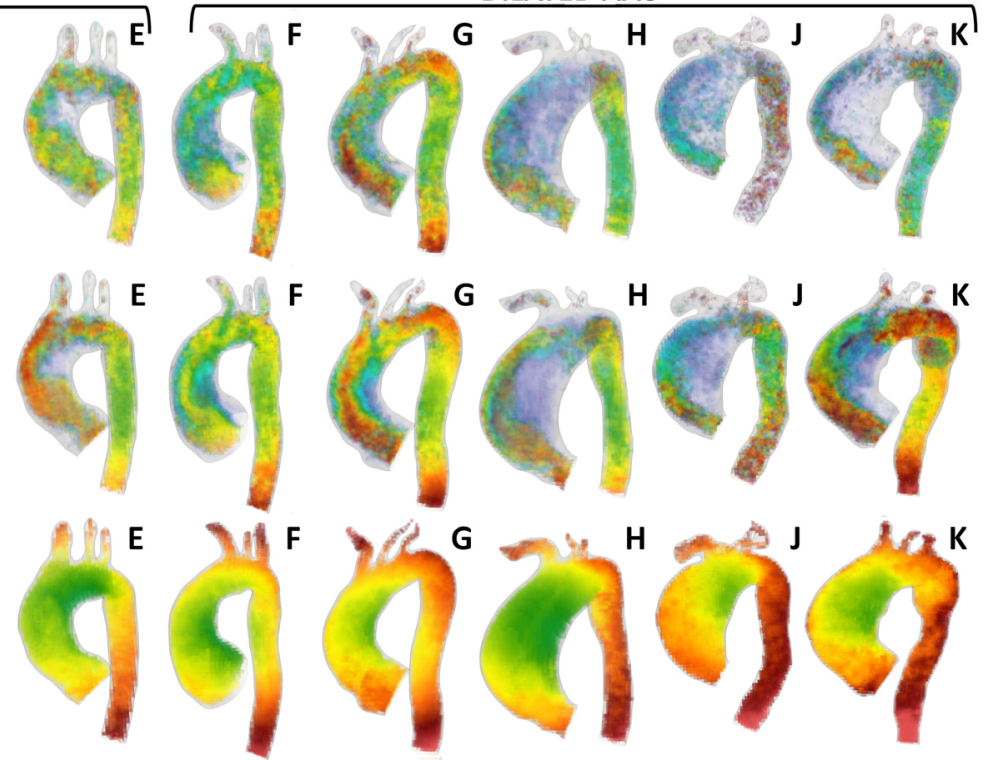

FIGURE 4. Volumetric maps of $h A E D$ for IVI (top row), $V_{\text {ax }}$ (middle row) and $v_{\text {sc }}$ (bottom row) CNs.

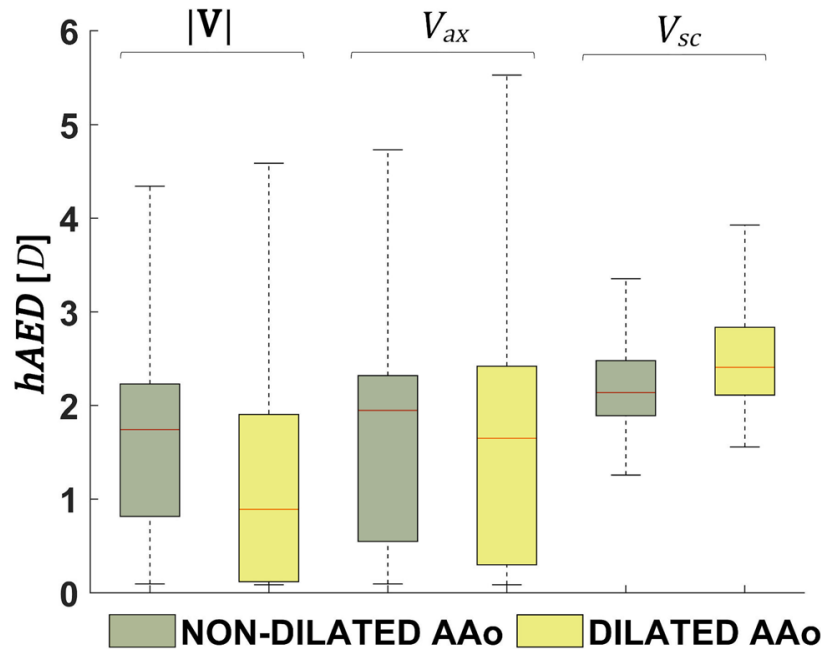

FIGURE 5. Effect of AAo dilation on the distributions of $h A E D$ values for IVI, $V_{\text {ax }}$ and $V_{\text {sc }} \mathrm{CNs}$. The median is indicated by the red line, the box indicates the interquartile range and the whiskers indicate the extreme values of the distribution.

(3.73- and 3.63-links path, in dilated and non-dilated patients, respectively, Table 2). Overall, the topological separation of the $V_{\text {sc }}$ networks is not sensitive to AAo dilation (1.53- and 1.51-links path, in dilated and non-dilated patients, respectively, Table 2).

\section{Relationships Between Complex Networks Metrics and Clinical Parameters}

Significant associations emerge between the geometric clinical parameter $D_{\text {ratio }}$ and the CNs metrics. In detail, $D_{\text {ratio }}$ is negatively associated with $h A E D$ med-
TABLE 2. ASPL values for IVI, $V_{\mathrm{ax}}$ and $V_{\mathrm{sc}} \mathrm{CNs}$.

\begin{tabular}{llccc}
\hline & Patient & IVI ASPL & $V_{\mathrm{ax}}$ ASPL & $V_{\mathrm{sc}}$ ASPL \\
\hline \multirow{2}{*}{ Non-dilated AAo } & $\mathrm{A}$ & 1.94 & 1.97 & 1.49 \\
& $\mathrm{~B}$ & 3.05 & 3.96 & 1.51 \\
& $\mathrm{C}$ & 3.01 & 3.63 & 1.54 \\
& $\mathrm{D}$ & 3.41 & 2.83 & 1.47 \\
& $\mathrm{E}$ & 3.33 & 3.66 & 1.51 \\
Dilated AAo & Median & 3.05 & 3.63 & 1.51 \\
& $\mathrm{~F}$ & 2.97 & 2.97 & 1.55 \\
& $\mathrm{G}$ & 3.30 & 3.03 & 1.53 \\
& $\mathrm{H}$ & 4.83 & 4.76 & 1.42 \\
& $\mathrm{~J}$ & 5.78 & 5.17 & 1.53 \\
& $\mathrm{~K}$ & 5.75 & 3.73 & 1.54 \\
& Median & 4.83 & 3.73 & 1.53 \\
\hline
\end{tabular}

ian values characterizing both $|\mathbf{V}|$ and $V_{\text {ax }}$ networks ( $p=0.020$ and $p=0.004$, respectively), suggesting a role for $D_{\text {ratio }}$ in disrupting the spatiotemporal hemodynamic similarity of $|\mathbf{V}|$ and $V_{\text {ax }}$ waveforms (Fig. 6). The results obtained considering the anatomical distances in the networks are confirmed by the topological analysis: a positive association emerges between $D_{\text {ratio }}$ and $A S P L$ values characterizing both $|\mathbf{V}|$ and $V_{\text {ax }}$ networks ( $p=0.020$ and $p=0.002$, respectively), suggesting that the aortic dilation breaks the correlation between $|\mathbf{V}|$ and $V_{\text {ax }}$ time-histories, increasing topological separation between the measured voxelbased velocity quantities.

No significant associations emerge between $D_{\text {ratio }}$ and the $h D C$ median values of the $|\mathbf{V}|$, and $V_{\text {ax }}$ networks (Fig. 6). No significant associations emerge 
- NON-DILATED AAo - DILATED AAo
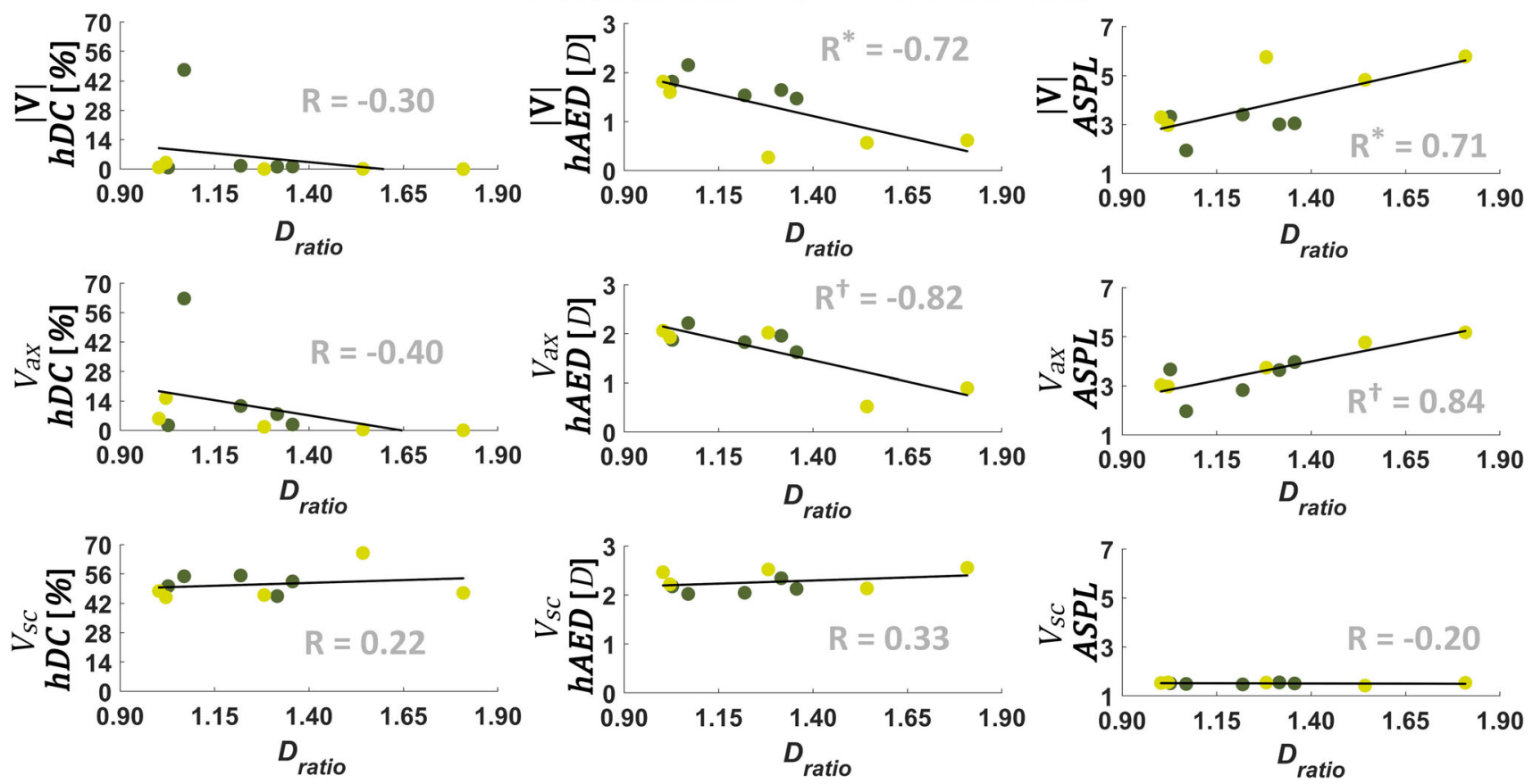

FIGURE 6. Associations between the geometric clinical indicator $D_{\text {ratio }}$ and CNs metrics $h D C$ (left column), $h A E D$ (middle column), and $A S P L$ (right column) for IVI (top row), $V_{\text {ax }}$ (middle row) and $V_{\text {sc }}$ (bottom row) CNs. For each CN, $h D C$ and $h A E D$ are expressed as the median value of all voxels. $R$ : Pearson's correlation coefficient $\left({ }^{\star} p<0.05,{ }^{\dagger} p<0.01\right)$.

between $D_{\text {ratio }}$ and $V_{\text {sc }}$ network metrics, a consequence of the low dispersion of the latter (median $h D C$ range $=[44.9 \%, 66.0 \%], A S P L$ range $=[1.42,1.55]$, median $h A E D$ range $=[2.02 D, 2.56 D]$, Fig. 6).

When considering the hemodynamic clinical parameters, a significant (albeit moderate) positive association emerges between blood flow $\mathrm{KE}$ at peak systole and $h D C$ median values characterizing the $|\mathbf{V}|$ networks $(p=0.04)$, likely driven by the high $h D C$ median value of patient A (rightmost point in Fig. 7, upper panel, resulting from the high $h D C$ values in the whole domain as depicted in the $h D C$ volumetric map of Fig. 3). A negative (albeit moderate) association emerges between KE at peak systole and $A S P L$ values characterizing the $|\mathbf{V}|$ networks ( $p=0.04$, Fig. 7). Taken together, these results suggest that high $\mathrm{KE}_{\text {peak }}$ values might contribute to increase the spatiotemporal homogeneity and topological length of correlation persistence of the velocity vector field, in aorta. Neither the other investigated hemodynamic clinical parameters nor the centerline-based aortic geometric attributes $\bar{\kappa}$ and $\bar{\tau}$ impact the spatiotemporal heterogeneity of phase velocity data, as no significant correlations emerge with CNs metrics (Figures S2-S7 of the Supplementary Data).
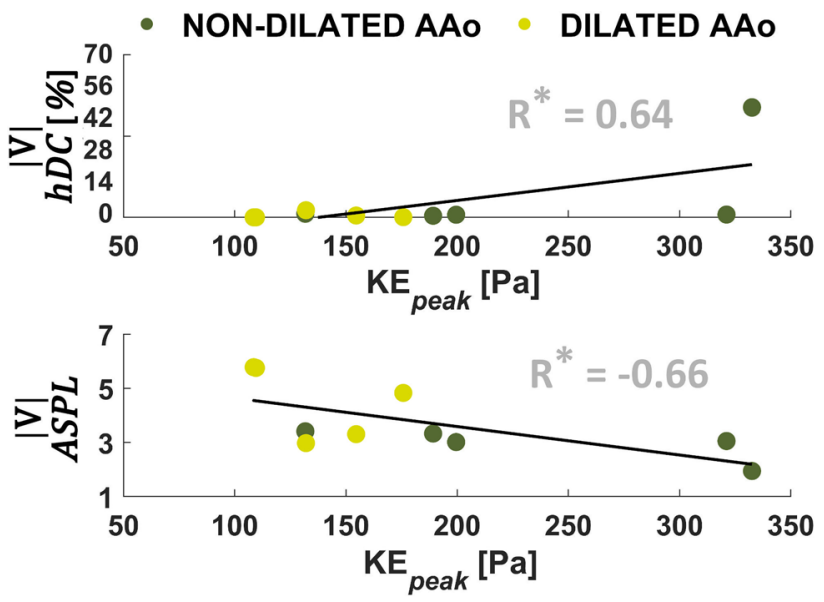

FIGURE 7. Associations of blood flow kinetic energy at the systolic peak (KE $\mathrm{K}_{\text {pak }}$ ) with $h D C$ (top) and ASPL (bottom) for IVI CNs. Here $h D C$ is expressed as the median value of all voxels. $R$ Pearson's correlation coefficient $\left({ }^{*} p<0.05\right.$, $p<0.01$ ).

\section{DISCUSSION}

The onset/progression of AAo wall dilation is the result of a complex multifactorial process in which local disturbed aortic hemodynamics plays a relevant role. $^{6,13,43}$ In recent years, 4D flow MRI has been frequently employed for aortic flow visualization and/ or characterization, aiming at elucidating the link 
between hemodynamic alterations and AAo disease in vivo. ${ }^{14,25,43}$

Stimulated by the need for interpretation of the intricate 4D aortic hemodynamics, and the identification of hemodynamic indicators/predictors of vascular disease to be adopted in a clinical framework, the current approach does not limit its focus on instantaneous snapshots or time- and space-average quantities, but takes into account the information embedded in the dynamical evolution of the aortic hemodynamics. To extend and deepen the in vivo characterization of hemodynamic complexity in the human aorta, the present study aims at investigating the spatiotemporal heterogeneity of large-scale aortic fluid structures by applying for the first time the Complex Networks theory to in vivo measured velocity data belonging to a 4D flow MRI dataset of human aortas with and without AAo dilation. In doing that, the CNs approach captures the large-scale "coherent flow structures" by implementing their definition as structures over which one fundamental macroscopic quantity (in this case, a velocity component or its magnitude) exhibits significant correlation with itself over a range of space and/or time significantly larger than the smallest scales of flow. ${ }^{42}$ The here-proposed CNs approach has the advantage of adding quantitative information to the detected coherent flow structures, represented by the anatomic and topological length of persistence of the correlation between the dynamical evolutions of the investigated quantities.

\section{CNs-Based Analysis of Aortic Flow Spatiotemporal Heterogeneity}

Among the main findings, it is here reported that most of the patients (with the exception of non-dilated patient A) present with large spatiotemporal heterogeneity of both $|\mathbf{V}|$ and $V_{\text {ax }}$ time-histories along the cardiac cycle, as the corresponding networks are characterized by very sparse connections and low degree of similarity (low $h D C$ ) between waveforms (Fig. 3). The distinguishable topologically isolated flow structures ( $h D C$ close to zero) identifiable by visual inspection at the AAo inner wall in the $V_{\text {ax }}$ networks of all investigated patients (Fig. 3) can be explained by the presence of large-scale flow recirculation patterns. ${ }^{19,31,46}$ Such flow features have been associated to (1) intimal lipid accumulation ${ }^{22}$ in murine and rabbit thoracic aorta, and (2) atherosclerotic and thrombotic biological markers in an arterial replication platform. ${ }^{32}$ As explained in Fig. 8 for a representative case, the marked flow reversal is highlighted by $V_{\text {ax }}$ waveforms with negative values, which are likely to be anti-correlated with $V_{\text {ax }}$ waveforms mainly aligned with the forward flow direction, resulting in negative $\mathrm{R}_{i j}^{a x}$ values (Figure $\mathrm{S} 1$ ). Interestingly, the symmetry of the $V_{\text {sc }}$ correlation distribution around zero for all patients (Figure S1 of the Supplementary Data), taken together with the dense patterns of connections (higher $h D C$ ) in $V_{\mathrm{sc}} \mathrm{CNs}$, hints at the presence of a Dean-like secondary flow organization. ${ }^{35}$ These findings, while preliminary, suggest that the aortic secondary flow patterns are more homogeneous than velocity magnitude and axial flow.

\section{The Impact of AAo Dilation on the Persistence Length of Correlation in Velocity Data}

The analysis of the spatial persistence of the correlation of aortic large-scale flow structures confirms the spatiotemporal heterogeneity of velocity data highlighted by $h D C$ (Fig. 3). Of relevance, the anatomic length of persistence of the correlation $h A E D$ in $V_{\mathrm{sc}}$ networks is higher than $|\mathbf{V}|$ and $V_{\text {ax }}$ networks (Figs. 4 and 5). In detail, the shape of $V_{\text {sc }}$ time-histories presents with a high level of similarity at anatomical distances longer than $2 \mathrm{D}$, a feature common both to dilated and non-dilated patients, while the similarity between $|\mathbf{V}|$ and $V_{\text {ax }}$ time-histories is bounded below $2 D$ (Fig. 5). The topological length of persistence of the correlation is also longer for $V_{\text {sc }}$ than $|\mathbf{V}|$ and $V_{\text {ax }}$ networks (as highlighted by $A S P L$ values in Table 2).

The combined picture provided by the calculation of $h A E D$ and $A S P L$ metrics suggests a different action for the AAo dilation on aortic velocity: a larger AAo dilation disrupts the (anatomical and topological) length of persistence of the similarity of both $|\mathbf{V}|$ and $V_{\mathrm{ax}}$ waveforms, while preserving more the spatiotemporal similarity of $V_{\mathrm{sc}}$ waveforms. These findings are in line with a recent study conducted on a dataset of computational hemodynamics models of healthy human carotid bifurcations, ${ }^{8}$ where a similar relation between the spatiotemporal heterogeneity of helical flow structures and a geometric indicator of carotid bulb expansion was reported.

The regression analysis conducted on CNs distance metrics and the geometric clinically measurable parameter $D_{\text {ratio }}$ corroborates the hypothesis of an impact of AAo dilation on blood velocity. As for the $V_{\mathrm{sc}}$ networks, no significant association emerges between the aortic $D_{\text {ratio }}$ and the network distance metrics $h A E D$ and $A S P L$. In other terms, the $V_{\mathrm{sc}}$ networks are more compact, less sensitive to AAo dilation than $|\mathbf{V}|$ and $V_{\text {ax }}$ networks.

On the contrary, from the analysis of $|\mathbf{V}|$ and $V_{\text {ax }}$ networks it emerges that the aortic $D_{\text {ratio }}$ is negatively correlated with hAED ( $\mathrm{R}=-0.72, p<0.05$ and $\mathrm{R}=-0.82, p<0.01$, respectively, Fig. 6) and positively correlated with $A S P L(\mathrm{R}=0.71, p<0.05$ and 


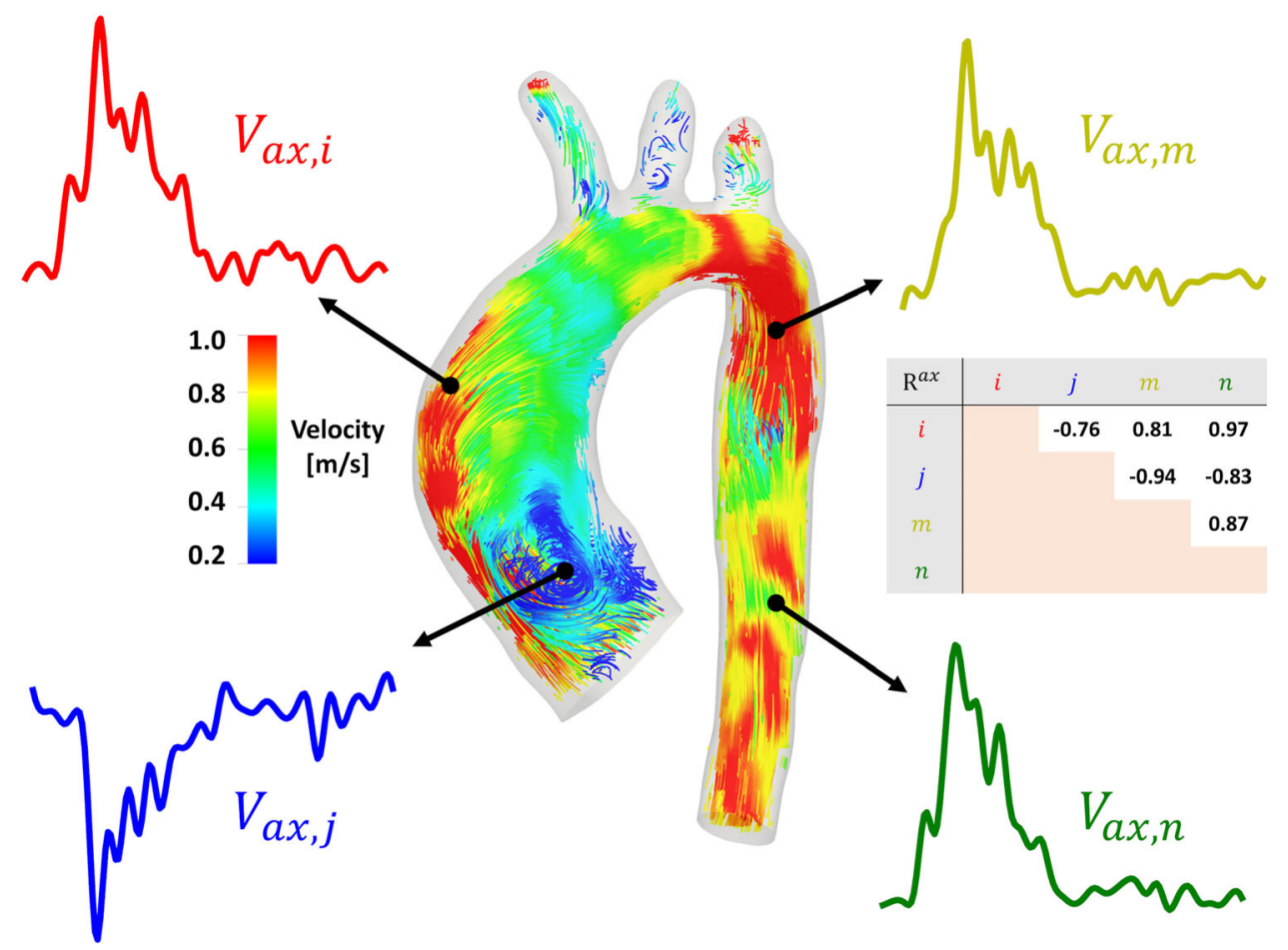

FIGURE 8. Explanatory example of differently correlated pairs of $V_{a x}$ time-histories in a representative case. Regions of flow reversal, like the inner AAo, are characterized by $V_{\mathrm{ax}}$ waveforms with negative values ( $V_{\mathrm{ax}, j}$ in the Figure), which are anti-correlated (negative $\mathrm{R}^{\mathrm{ax}}$ values) with $V_{\mathrm{ax}, j}$ waveforms mainly aligned with the forward flow direction ( $V_{\mathrm{ax}, i}, V_{\mathrm{ax}, m}$ and $V_{\mathrm{ax}, n}$ in the Figure). $V_{\mathrm{ax}}$ waveforms shown in the Figure were interpolated using a higher number of time points for visualization purposes.

$\mathrm{R}=0.84, p<0.01$, respectively, Fig. 6). From a fluid mechanics viewpoint, the present analysis demonstrates that AAo dilation, promoting the development of flow disturbances, has a detrimental effect on the spatiotemporal coherence of $|\mathbf{V}|$ and $V_{\text {ax }}$ waveforms in the aorta. What emerges from the in vivo CNs-based quantitative analysis agrees with previous semi-quantitative studies reporting of an association (1) between age, AAo diameter and the presence of large vortices in aorta, ${ }^{18}$ and (2) between the presence and strength of vortical and helical flow patterns and AAo diameter and AAo/DAo diameter ratio. ${ }^{6}$ Interestingly, and consistently with the findings of this in vivo analysis, a very recent study integrating $\mathrm{CNs}$ with computational hemodynamics simulations suggests that the length of correlation persistence in forward flow could be shorter in the presence of AAo dilation, compared to the healthy ascending aorta. ${ }^{13}$ The emergent picture leads to presume that the physiological spatiotemporal coherence of large-scale forward flow could be progressively compromised in the presence of increasing AAo dilation. The capability of the $\mathrm{CNs}$ analysis to underscore intravascular flow spatiotemporal heterogeneity and disturbed flow features like flow separation and recirculation, increased velocity jets, highly rotational flow, allows a deeper understanding of the interaction of these intravascular fluid structures with the vascular wall, and ultimately near-wall biological transport and endothelial-cell mediated pathways involved in vascular remodeling and dilation. $^{30,33,35,40,49}$ In addition to that, given the importance of intravascular flow features in terms of flow energetics, the correlation persistence, measured by $\mathrm{CNs}$, as well as the amount of spatiotemporal heterogeneity in the flow field, is expected to have an impact in the ventricular work.

A main limitation that could potentially weaken the findings of this study is the choice of the correlation threshold used to build the CNs. Consistently with previous CNs-based in silico studies, ${ }^{7,8,13}$ the $\mathrm{CNs}$ threshold for a specific hemodynamic quantity was set equal to the median value of the correlation coefficients distribution obtained from a dataset of computational hemodynamic models of healthy aortas. Further studies on a 4D flow MRI dataset of healthy subjects could be useful to investigate the robustness of the selected thresholds. Although the noise typically affecting 4D flow MRI acquisitions could cause an underestimation of the correlations between velocity waveforms, the pattern of connection between nodes resulting from the threshold-based approach adopted in this study to build the $\mathrm{CN}$ is expected to be marginally influenced by the use of in vivo data as acquired. In addition to that, the systolic phase, which is ex- 
pected to drive the correlations, is characterized by a higher signal-to-noise ratio than the diastolic phase, therefore dampening the impact of noise on the correlation distribution. Another limitation of this study lies in its cross-sectional nature, which does not allow to draw any conclusion about the potential of the approach as an in vivo tool for risk prediction, for which longitudinal studies are needed. However, this was intended as an exploratory study aiming at testing the ability of the CNs to characterize in vivo complex aortic flows and their link to vascular disease. All patients enrolled for this study presented with aortic valvular dysfunctions (from mild to severe), and two of them with BAV (Table 1), thus preventing a robust comparison between valve-mediated hemodynamics in BAV vs. TAV groups. Moreover, the scarce stratification as well as the small sample size could have polarized the regression analysis results involving $h D C$ and influenced the associations between $\mathrm{CNs}$ metrics and the other investigated clinical hemodynamic parameters and centerline-based geometric attributes. In addition to that, based on the scatter plots in Figs. 6, 7 and in the Supplementary Data, non-dilated patient $\mathrm{A}$ appears as an outlier/leverage point in relation of the $h D C$ metric for the $|\mathbf{V}|$ and $V_{\text {ax }}$ networks. However, when removing patient $\mathrm{A}$ from the regression analysis, the observed statistical significance (Fig. 6) of the associations between CNs distance metrics and $D_{\text {ratio }}$ is preserved, whereas the correlations with $\mathrm{KE}_{\text {peak }}$ in the $|\mathbf{V}| \mathrm{CNs}$ (Fig. 7) become non statistically relevant. Therefore, further investigations with larger and better stratified datasets are needed to confirm the present findings on the spatiotemporal heterogeneity of large-scale flow features.

In conclusion, in this study the CNs theory was applied to in vivo velocity data from a 4D flow MRI dataset of human aortas to (1) investigate the spatiotemporal heterogeneity of large-scale aortic flow structures and (2) assess the existence of possible associations between $\mathrm{CNs}$ metrics and ascending aortic dilation. Results showed that velocity magnitude and through-plane (axial) velocity structures are characterized by a larger spatiotemporal heterogeneity than in-plane (secondary) flow structures. Moreover, an increasing AAo dilation disrupts the correlation in forward flow reducing the correlation persistence length, while preserving the spatiotemporal homogeneity of secondary flows. The here presented approach for obtaining in vivo measurable information on aortic hemodynamics by integrating 4D flow MRI and $\mathrm{CNs}$ in a clinical framework shows a strong potential as a tool for visualization and quantification of complex cardiovascular flows, and the use of CNs distance metrics may allow a finer risk stratification of AAo disease.

\section{FUNDING}

Open access funding provided by Politecnico di Torino within the CRUI-CARE Agreement.

\section{SUPPLEMENTARY INFORMATION}

The online version of this article (https://doi.org/10. 1007/s10439-021-02798-9).

\section{ACKNOWLEDGEMENTS}

Guala A. has received funding from the Spanish Ministry of Science, Innovation and Universities (IJC2018-037349-I). Gallo D. and Morbiducci U. were supported by MIUR FISR-FISR2019_03221 CECOMES. The Authors thank Dr. Giovanna Rizzo for the precious insights.

\section{OPEN ACCESS}

This article is licensed under a Creative Commons Attribution 4.0 International License, which permits use, sharing, adaptation, distribution and reproduction in any medium or format, as long as you give appropriate credit to the original author(s) and the source, provide a link to the Creative Commons licence, and indicate if changes were made. The images or other third party material in this article are included in the article's Creative Commons licence, unless indicated otherwise in a credit line to the material. If material is not included in the article's Creative Commons licence and your intended use is not permitted by statutory regulation or exceeds the permitted use, you will need to obtain permission directly from the copyright holder. To view a copy of this licence, visit http://crea tivecommons.org/licenses/by/4.0/.

\section{REFERENCES}

${ }^{1}$ Antiga, L., M. Piccinelli, L. Botti, B. Ene-Iordache, A.
Remuzzi, and D. A. Steinman. An image-based modeling
framework for patient-specific computational hemody-
namics. Med. Biol. Eng. Comput. 46:1097, 2008.
${ }^{2}$ Bargiotas, I., E. Mousseaux, W.-C. Yu, B. A. Venkatesh,
E. Bollache, A. de Cesare, J. A. C. Lima, A. Redheuil, and
N. Kachenoura. Estimation of aortic pulse wave transit
time in cardiovascular magnetic resonance using complex
wavelet cross-spectrum analysis. J. Cardiovasc. Magn.
Reson. Off. J. Soc. Cardiovasc. Magn. Reson. 17:65, 2015.
${ }^{3}$ Barker, A. J., M. Markl, J. Bürk, R. Lorenz, J. Bock, S.
Bauer, J. Schulz-Menger, and F. von Knobelsdorff-
Brenkenhoff. Bicuspid aortic valve is associated with alBMES 
tered wall shear stress in the ascending aorta. Circ. Cardiovasc. Imaging 5:457-466, 2012.

${ }^{4}$ Bieging, E. T., A. Frydrychowicz, A. Wentland, B. R. Landgraf, K. M. Johnson, O. Wieben, and C. J. François. In vivo three-dimensional MR wall shear stress estimation in ascending aortic dilatation. J. Magn. Reson. Imaging 33:589-597, 2011.

${ }^{5}$ Bogren, H. G., and M. H. Buonocore. 4D magnetic resonance velocity mapping of blood flow patterns in the aorta in young vs. elderly normal subjects. J. Magn. Reson. Imaging 10:861-869, 1999.

${ }^{6}$ Burk, J., P. Blanke, Z. Stankovic, A. Barker, M. Russe, J. Geiger, A. Frydrychowicz, M. Langer, and M. Markl. Evaluation of 3D blood flow patterns and wall shear stress in the normal and dilated thoracic aorta using flow-sensitive 4D CMR. J. Cardiovasc. Magn. Reson. 14:84, 2012.

${ }^{7}$ Calò, K., G. De Nisco, D. Gallo, C. Chiastra, A. Hoogendoorn, D. A. Steinman, S. Scarsoglio, J. J. Wentzel, and U. Morbiducci. Exploring wall shear stress spatiotemporal heterogeneity in coronary arteries combining correlation-based analysis and complex networks with computational hemodynamics. Proc. Inst. Mech. Eng. Part H 234:1209-1222, 2020.

${ }^{8}$ Calò, K., D. Gallo, D. A. Steinman, V. Mazzi, S. Scarsoglio, L. Ridolfi, and U. Morbiducci. Spatiotemporal hemodynamic complexity in carotid arteries: an integrated computational hemodynamics \& complex networks-based approach. IEEE Trans. Biomed. Eng. 67:1841-1853, 2020. ${ }^{9}$ Campens, L., L. Demulier, K. De Groote, K. Vandekerckhove, D. De Wolf, M. J. Roman, R. B. Devereux, A. De Paepe, and J. De Backer. Reference values for echocardiographic assessment of the diameter of the aortic root and ascending aorta spanning all age categories. Am. J. Cardiol. 114:914-920, 2014.

${ }^{10} \mathrm{Chau}, \mathrm{K}$. H., and J. A. Elefteriades. Natural history of thoracic aortic aneurysms: size matters, plus moving beyond size. Prog. Cardiovasc. Dis. 56:74-80, 2013.

${ }^{11}$ Condemi, F., S. Campisi, M. Viallon, P. Croisille, J.-F. Fuzelier, and S. Avril. Ascending thoracic aorta aneurysm repair induces positive hemodynamic outcomes in a patient with unchanged bicuspid aortic valve. J. Biomech. 81:145148, 2018.

${ }^{12}$ Davis, A. E., A. J. Lewandowski, C. J. Holloway, N. A. B. Ntusi, R. Banerjee, R. Nethononda, A. Pitcher, J. M. Francis, S. G. Myerson, P. Leeson, T. Donovan, S. Neubauer, and O. J. Rider. Observational study of regional aortic size referenced to body size: production of a cardiovascular magnetic resonance nomogram. J. Cardiovasc. Magn. Reson. Off. J. Soc. Cardiovasc. Magn. Reson. 16:9, 2014.

${ }^{13}$ De Nisco, G., P. Tasso, K. Calò, V. Mazzi, D. Gallo, F. Condemi, S. Farzaneh, S. Avril, and U. Morbiducci. Deciphering ascending thoracic aortic aneurysm hemodynamics in relation to biomechanical properties. Med. Eng. Phys. 82:119-129, 2020.

${ }^{14}$ Dux-Santoy, L., A. Guala, G. Teixidó-Turà, A. RuizMuñoz, G. Maldonado, N. Villalva, L. Galian, F. Valente, L. Gutiérrez, T. González-Alujas, A. Sao-Avilés, K. M. Johnson, O. Wieben, M. Huguet, D. García-Dorado, A. Evangelista, and J. F. Rodríguez-Palomares. Increased rotational flow in the proximal aortic arch is associated with its dilation in bicuspid aortic valve disease. Eur. Hear. J. - Cardiovasc. Imaging 20:1407-1417, 2019.
${ }^{15}$ Elefteriades, J. A., and E. A. Farkas. Thoracic aortic aneurysm clinically pertinent controversies and uncertainties. J. Am. Coll. Cardiol. 55:841-857, 2010.

${ }^{16}$ Emendi, M., F. Sturla, R. P. Ghosh, M. Bianchi, F. Piatti, F. R. Pluchinotta, D. Giese, M. Lombardi, A. Redaelli, and D. Bluestein. Patient-Specific Bicuspid Aortic Valve Biomechanics: a Magnetic Resonance Imaging Integrated Fluid-Structure Interaction Approach. Ann. Biomed. Eng. 49:627-641, 2021.

${ }^{17}$ Erbel, R., V. Aboyans, C. Boileau, E. Bossone, R. Di Bartolomeo, H. Eggebrecht, A. Evangelista, V. Falk, H. Frank, O. Gaemperli, M. Grabenwoger, A. Haverich, B. Iung, A. J. Manolis, F. Meijboom, C. A. Nienaber, M. Roffi, H. Rousseau, U. Sechtem, P. A. Sirnes, R. S. von Allmen, and C. J. M. Vrints. 2014 ESC Guidelines on the diagnosis and treatment of aortic diseases: document covering acute and chronic aortic diseases of the thoracic and abdominal aorta of the adult: the Task Force for the Diagnosis and Treatment of Aortic Diseases of the European. Eur. Heart J. 35:2873-2926, 2014.

${ }^{18}$ Frydrychowicz, A., A. Berger, A. M. Del Rio, M. F. Russe, J. Bock, A. Harloff, and M. Markl. Interdependencies of aortic arch secondary flow patterns, geometry, and age analysed by 4-dimensional phase contrast magnetic resonance imaging at 3 Tesla. Eur. Radiol. 22:1122-1130, 2012.

${ }^{19}$ Frydrychowicz, A., A. Berger, M. F. Russe, A. F. Stalder, A. Harloff, S. Dittrich, J. Hennig, M. Langer, and M. Markl. Time-resolved magnetic resonance angiography and flow-sensitive 4-dimensional magnetic resonance imaging at 3 Tesla for blood flow and wall shear stress analysis. $J$. Thorac. Cardiovasc. Surg. 136:400-407, 2008.

${ }^{20}$ Gallo, D., G. De Santis, F. Negri, D. Tresoldi, R. Ponzini, D. Massai, M. A. Deriu, P. Segers, B. Verhegghe, G. Rizzo, and U. Morbiducci. On the use of in vivo measured flow rates as boundary conditions for image-based hemodynamic models of the human aorta: implications for indicators of abnormal flow. Ann. Biomed. Eng. 40:729-741, 2012.

${ }^{21}$ Gallo, D., D. A. Steinman, P. B. Bijari, and U. Morbiducci. Helical flow in carotid bifurcation as surrogate marker of exposure to disturbed shear. J. Biomech. 45:2398-2404, 2012

${ }^{22}$ Gimbrone, M. A. The Gordon Wilson lecture. Understanding vascular endothelium: a pilgrim's progress: Endothelial dysfunction, biomechanical forces and the pathobiology of atherosclerosis. 2010.

${ }^{23}$ Guala, A., J. Rodriguez-Palomares, L. Dux-Santoy, G. Teixido-Tura, G. Maldonado, L. Galian, M. Huguet, F. Valente, L. Gutiérrez, T. González-Alujas, K. M. Johnson, O. Wieben, A. Sao Avilés, D. Garcia-Dorado, and A. Evangelista. Influence of aortic dilation on the regional aortic stiffness of bicuspid aortic valve assessed by 4-dimensional flow cardiac magnetic resonance: comparison with marfan syndrome and degenerative aortic aneurysm. JACC 12:1020-1029, 2019.

${ }^{24}$ Guzzardi, D. G., A. J. Barker, P. van Ooij, S. C. Malaisrie, J. J. Puthumana, D. D. Belke, H. E. M. Mewhort, D. A. Svystonyuk, S. Kang, S. Verma, J. Collins, J. Carr, R. O. Bonow, M. Markl, J. D. Thomas, P. M. McCarthy, and P. W. M. Fedak. Valve-related hemodynamics mediate human bicuspid aortopathy: insights from wall shear stress mapping. J. Am. Coll. Cardiol. 66:892-900, 2015.

${ }^{25}$ Hassanabad, A. F., F. Burns, M. S. Bristow, C. Lydell, A. G. Howarth, B. Heydari, X. Gao, P. W. M. Fedak, J. A. White, and J. Garcia. Pressure drop mapping using 4D flow 
MRI in patients with bicuspid aortic valve disease: A novel marker of valvular obstruction. Magn. Reson. Imaging 65:175-182, 2020.

${ }^{26}$ Hope, M. D., T. A. Hope, A. K. Meadows, K. G. Ordovas, T. H. Urbania, M. T. Alley, and C. B. Higgins. Bicuspid aortic valve: four-dimensional MR evaluation of ascending aortic systolic flow patterns. Radiology 255:53-61, 2010.

${ }^{27}$ Isselbacher, E. M. Thoracic and abdominal aortic aneurysms. Circulation 111:816-828, 2005.

${ }^{28}$ Johnson, E. M. I., J. A. Heller, F. G. Vicente, R. Sarnari, D. Gordon, P. M. McCarthy, A. J. Barker, M. Etemadi, and M. Markl. Detecting aortic valve-induced abnormal flow with seismocardiography and cardiac MRI. Ann. Biomed. Eng. 48:1779-1792, 2020.

${ }^{29}$ Johnson, K. M., D. P. Lum, P. A. Turski, W. F. Block, C. A. Mistretta, and O. Wieben. Improved 3D phase contrast MRI with off-resonance corrected dual echo VIPR. Magn. Reson. Med. 60:1329-1336, 2008.

${ }^{30}$ Liu, X., A. Sun, Y. Fan, and X. Deng. Physiological significance of helical flow in the arterial system and its potential clinical applications. Ann. Biomed. Eng. 43:3-15, 2015.

${ }^{31}$ Mark1, M., M. T. Draney, M. D. Hope, J. M. Levin, F. P. Chan, M. T. Alley, N. J. Pelc, and R. J. Herfkens. Timeresolved 3-dimensional velocity mapping in the thoracic aorta: visualization of 3-directional blood flow patterns in healthy volunteers and patients. J. Comput. Assist. Tomogr. 28:459-468, 2004.

${ }^{32}$ Martorell, J., P. Santomá, K. Kolandaivelu, V. B. Kolachalama, P. Melgar-Lesmes, J. J. Molins, L. Garcia, E. R. Edelman, and M. Balcells. Extent of flow recirculation governs expression of atherosclerotic and thrombotic biomarkers in arterial bifurcations. Cardiovasc. Res. 103:37-46, 2014.

${ }^{33}$ Morbiducci, U., D. Gallo, S. Cristofanelli, R. Ponzini, M. A. Deriu, G. Rizzo, and D. A. Steinman. A rational approach to defining principal axes of multidirectional wall shear stress in realistic vascular geometries, with application to the study of the influence of helical flow on wall shear stress directionality in aorta. J. Biomech. 48:899-906, 2015.

${ }^{34}$ Morbiducci, U., R. Ponzini, D. Gallo, C. Bignardi, and G. Rizzo. Inflow boundary conditions for image-based computational hemodynamics: impact of idealized versus measured velocity profiles in the human aorta. J. Biomech. 46:102-109, 2013.

${ }^{35}$ Morbiducci, U., R. Ponzini, G. Rizzo, M. Cadioli, A. Esposito, F. M. Montevecchi, and A. Redaelli. Mechanistic insight into the physiological relevance of helical blood flow in the human aorta: an in vivo study. Biomech. Model. Mechanobiol. 10:339-355, 2011.

${ }^{36}$ Muraru, D., J. Bidviene, G. Cavalli, A. Cavaliere, and L. P. Badano. Tricuspid regurgitation in a patient with ascending aorta aneurysm. Eur. Heart J. Cardiovasc. Imaging 17:1435, 2016.

${ }^{37}$ Newman, M. Networks. Oxford: Oxford University Press, 2018.

${ }^{38}$ Pape, L. A., T. T. Tsai, E. M. Isselbacher, J. K. Oh, P. T. Ogara, A. Evangelista, R. Fattori, G. Meinhardt, S. Trimarchi, E. Bossone, T. Suzuki, J. V. Cooper, J. B. Froehlich, C. A. Nienaber, and K. A. Eagle. Aortic diameter > or $=5.5 \mathrm{~cm}$ is not a good predictor of type A aortic dis- section: observations from the International Registry of Acute Aortic Dissection (IRAD). Circulation 116:11201127, 2007.

${ }^{39}$ Pasta, S., V. Agnese, A. Gallo, F. Cosentino, M. Di Giuseppe, G. Gentile, G. M. Raffa, J. F. Maalouf, H. I. Michelena, and D. Bellavia. Shear stress and aortic strain associations with biomarkers of ascending thoracic aortic aneurysm. Ann. Thorac. Surg. 110:1595-1604, 2020.

${ }^{40}$ Pasta, S., G. Gentile, G. M. Raffa, D. Bellavia, G. Chiarello, R. Liotta, A. Luca, C. Scardulla, and M. Pilato. In Silico shear and intramural stresses are linked to aortic valve morphology in dilated ascending aorta. Eur. J. Vasc. Endovasc. Surg. 54:254-263, 2017.

${ }^{41}$ Piccinelli, M., A. Veneziani, D. A. Steinman, A. Remuzzi, and L. Antiga. A framework for geometric analysis of vascular structures: application to cerebral aneurysms. IEEE Trans. Med. Imaging 28:1141-1155, 2009.

${ }^{42}$ Robinson, S. K. Coherent motions in the turbulent boundary layer. Annu. Rev. Fluid Mech. 23:601-639, 1991.

${ }^{43}$ Rodríguez-Palomares, J. F., L. Dux-Santoy, A. Guala, R. Kale, G. Maldonado, G. Teixidó-Turà, L. Galian, M. Huguet, F. Valente, L. Gutiérrez, T. González-Alujas, K. M. Johnson, O. Wieben, D. García-Dorado, and A. Evangelista. Aortic flow patterns and wall shear stress maps by 4D-flow cardiovascular magnetic resonance in the assessment of aortic dilatation in bicuspid aortic valve disease. J. Cardiovasc. Magn. Reson. 20:28, 2018.

${ }^{44}$ Salmasi, M., O. A. Jarral, S. Pirola, S. Sasidharan, J. Pepper, A. Oo, J. Moore, Jr, X. Y. Xu, and T. Athanasiou. In-vivo blood flow parameters can predict at-risk aortic aneurysms and dissection: a comprehensive biomechanics model. Eur. Heart J. 41:46-2339, 2020.

${ }^{45}$ Sigovan, M., M. D. Hope, P. Dyverfeldt, and D. Saloner. Comparison of four-dimensional flow parameters for quantification of flow eccentricity in the ascending aorta. $J$. Magn. Reson. Imaging 34:1226-1230, 2011.

${ }^{46}$ van Ooij, P., E. S. Farag, C. P. S. Blanken, A. J. Nederveen, M. Groenink, R. N. Planken, and S. M. Boekholdt. Fully quantitative mapping of abnormal aortic velocity and wall shear stress direction in patients with bicuspid aortic valves and repaired coarctation using 4D flow cardiovascular magnetic resonance. J. Cardiovasc. Magn. Reson. Off. J. Soc. Cardiovasc. Magn. Reson. 23:9, 2021.

${ }^{47}$ Volonghi, P., D. Tresoldi, M. Cadioli, A. M. Usuelli, R. Ponzini, U. Morbiducci, A. Esposito, and G. Rizzo. Automatic extraction of three-dimensional thoracic aorta geometric model from phase contrast MRI for morphometric and hemodynamic characterization. Magn. Reson. Med. 75:873-882, 2016.

${ }^{48}$ Wagenseil, J. E. Bio-chemo-mechanics of thoracic aortic aneurysms. Curr. Opin. Biomed. Eng. 5:50-57, 2018.

${ }^{49}$ Youssefi, P., A. Gomez, T. He, L. Anderson, N. Bunce, R. Sharma, C. A. Figueroa, and M. Jahangiri. Patient-specific computational fluid dynamics-assessment of aortic hemodynamics in a spectrum of aortic valve pathologies. $J$. Thorac. Cardiovasc. Surg. 153:8-20.e3, 2017.

Publisher's Note Springer Nature remains neutral with regard to jurisdictional claims in published maps and institutional affiliations. 